


\section{Cognitive dynamics}

$$
\text { 整 }
$$

BY: Luca Tateo

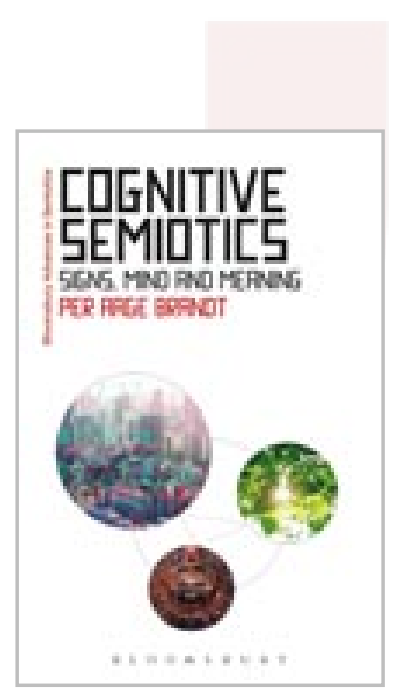

Brandt, Per Aage, 2020

\section{Cognitive Semiotics: Signs, Mind, and Meaning}

London: Bloomsbury Academic. 241 pp. $£ 95.00$ (HBK, ISBN 9781350143302, epub / Mobibook ISBN 9781350143326, pdf/ebook ISBN 9781350143319) ISBN 978-1-78406-678-9 (e-book), 206 p.

$\Pi$ he proliferation of disciplinary labels, distinctions, 1 borders, and hierarchies is an interesting semiotic phenomenon per se. In particular, the need to circumscribe a new semiotics field and then denoting it as transdisciplinary appears instead to be an exercise of politics. One cannot but fall into the paradox of any systemic organization, nicely described by Simmel a long time before:

By choosing two items from the undisturbed store of natural things in order to designate them as "separate," we have already related them to one another in our consciousness, we have emphasized these two together against whatever lies between them. And conversely, we can only sense those things to be related which we have previously somehow isolated from one another; things must first be separated from one ARTICLE INFO:

Volume: 06

Issue: 02:2020

Month: March

ISSN: 2459-2943

DOI: 10.18680/hss.2020.0032

Pages: 253-259

LiC.: CC BY-NC-ND 4.0 another in order to be together. (Simmel 1994: 5)

The field of Cognitive Semiotics emerged as a reaction to computationalism in cognitive sciences (Zlatev 2012). Any form of structural reductionism soon confronts the problem of meaning (Brandt 2020). In the introductory chapter of his seminal Acts of Meaning, Jerome Bruner, one of the founders of the so-called 'cognitive revolution,' attributes its partial failure to overlooking the human being as an active producer of meaning in favor of the modular brain idea as a mechanism of informa- 
tion processing. Hence, the need for a new holistic look at the meaning-making processes that characterize the human species. Simmel's paradox also applies to studying psychological functions as distinct submodules of the human mind. Once separated, cognition, volition, emotion, and body demand the development of a theory that puts them back together. The solution of cognitive semiotics is to replace the notion of structure and modularity with the idea of "architecture," i.e., a stable hierarchical organization of structures and functions whose form is not interchangeable. The modularity notion implies the possibility of recombining hierarchically equivalent modules without dramatically altering the whole's identity. As the prefabricated modules used in the building industry, one can recombine them as Lego bricks in different equivalent forms. Architecture has to do with the unique creation of 'patterns' through the hierarchical organization of elements. For instance, in an 18th-century neoclassical building's façade, the order and number of the columns and windows constitute the building's unique personality. If we modify one of its sub-parts, the whole system of proportions comes apart. It will become a different type of building.

\section{A cognitive architecture of meaning-making}

Per Brandt's book is quite literally built as a combination of architectural elements new chapters combined with republished, earlier works - aimed at presenting a general theory of how "representational meaning can be modeled" (Brandt 2020: 71). The central tenet is that the semiotic behavior of humans is not just an emergent property of embodied processes, but that meaning requires a form of representation, to the extent that meaning is "related both to the imaginary and to the experiential world" (Brandt 2020: 71). In the first part of his book, Brandt tries to provide a general model of the cognitive architecture that makes human meaning-making possible. He claims that, to link afferent (sensation) and efferent (action) behavior in the physical world, there must be a part of the circuit/ architecture which is situated on a different plane, enabling the appreciation and the planning of past and future actions, as well as those alternative courses of actions that will never appear in the physical world. Such mental architecture that operates in between afferent and efferent behavior has the form of an integrative cycle: "perceived forms $\rightarrow$ categorized objects $\rightarrow$ integrated situations [that Brandt calls "mental spaces"] $\rightarrow$ action-planning reflections $\rightarrow$ evaluating affects" (Brandt 2020: 73). This architecture allows the integration of qualia into objects, objects into categories, and a system of relations that can be called situations - in real or hypothetical forms. Conditions allow the creation of notions, abstract and generalizable representations, that finally lead to affects, i.e., the emotional appreciation of the experience.

The cognitive architecture's key element is mental space: a portion of meaning "that comes with an internal conceptual structure, a minimum of imagery, and a phenom- 
enological status as a scenario that can be referred to." (Brandt 2020: 83). The topical notion is the organization of objects into configurations according to principles that may come from the distal experience of culture, proximal phenomenological experience, or the production of fictional worlds. Meaning production occurs when humans compare, transfer, and blend the references of different mental spaces. In the second part of the book, Brandt provides several examples of using the meaning's mental architecture to analyze different kinds of texts (novels, poems, translations, numbers, money, etc.). The author's goal is to demonstrate that his architecture can account for the production of meaning in human activities, relating the embodied experience and the mental representation without necessarily conflating them into the monist notion of a non-representational physically-embodied emerging mind. His mental space model can map all the phenomenology of human meaning-making manifested in cultural products.

\section{Critical remarks}

Although Brandt provides several interesting examples, his claim of having developed a comprehensive theory of representational meaning, alternative to the other path towards universal semiosis, biosemiotics, seems too ambitious. Brandt's work remains within the limits of a semiotic of text, which of course, applies to different textual genres. Brandt's model is topological, not really dynamic, and processual. Indeed, his representation of the transformation between two meaning spaces, or the transformation of the reference due to the establishment of a new relationship between the spaces, does not account for the process of transformation. Brandt takes two repeated pictures of a building to observe the transformation of the architectural arrangement. Moreover, he presents two different buildings' images, showing how the architectural patterns have been placed in the two buildings. This is what he does, in effect, when he analyses two poems by Yeats and Woodsworth, respectively, where he discusses the imaginative process and the meaning spaces.

A repeated series of pictures cannot account for dynamism; it can be a two-dimensional representation of a process unfolding in time. Besides, a combinational model, such as Brandt's hierarchic architecture of qualia, objects, situations, evaluation, and affects, defines a topology but not the rules of transformation over time. Brandt's different graphic representations account for the relationships between the elements before and after a transformation, but not for the reconfiguration process that produced this transformation. In other words, the structural aspect of systems of oppositions / relations prevails over a dynamic view of parts/whole relationships. Brandt's model is quite useful in mapping different kinds of texts and showing how meaning is actively produced by creating mental spaces networks. Yet, I think it overlooks one crucial point of 
Peirce's universal theory of semiosis. Firstness is the encounter not with qualia but with the un-distinct flow of the world. The first relationship with the world is affective and physiognomic (Tateo 2018). We do not initially experience redness, softness, coldness, etc., and proceed to form objects and then relations, to finally decide or appreciate something about these objects in the context of their relationships. We primarily experience physiognomies, aesthetic configurations of elements, with which we relate affectively (Tateo 2018).

Distinctions emerge later, when affect and preferences attach to them: first, I experience $\mathrm{ABC}$ as a physiognomy. Subsequently, my relating to it produces a distinction $\mathrm{A}+\mathrm{B}+\mathrm{C}$, which is affectively charged. If $\mathrm{A}$ is distinct from $\mathrm{B}$ and $\mathrm{C}$, one must be better than another. Hence, this time within what Brandt calls 'mental space,' a new reconfiguration can emerge. As Simmel (1994) points out, we can experience distinction only between things that are related, and we can create relationships only between things that are distinct. Secondness and Thirdness are the conditions for meaning production. However, the exact lower and upper thresholds of meaning cannot be precisely located (Innis 2016). Before distinction-making, which is mainly socially guided through symbolic work, certain meaning-making forms precede the segmentation of experience or exceed it. Biosemiotics and aesthetics try to account for those phenomena, while cognitive semiotics seems not interested in them.

How does Brandt's model can be developed in a more dynamic-processual direction? For instance, let's take an example of his semiotic blending model from the book (figure 1, in Brandt 2020: 107).

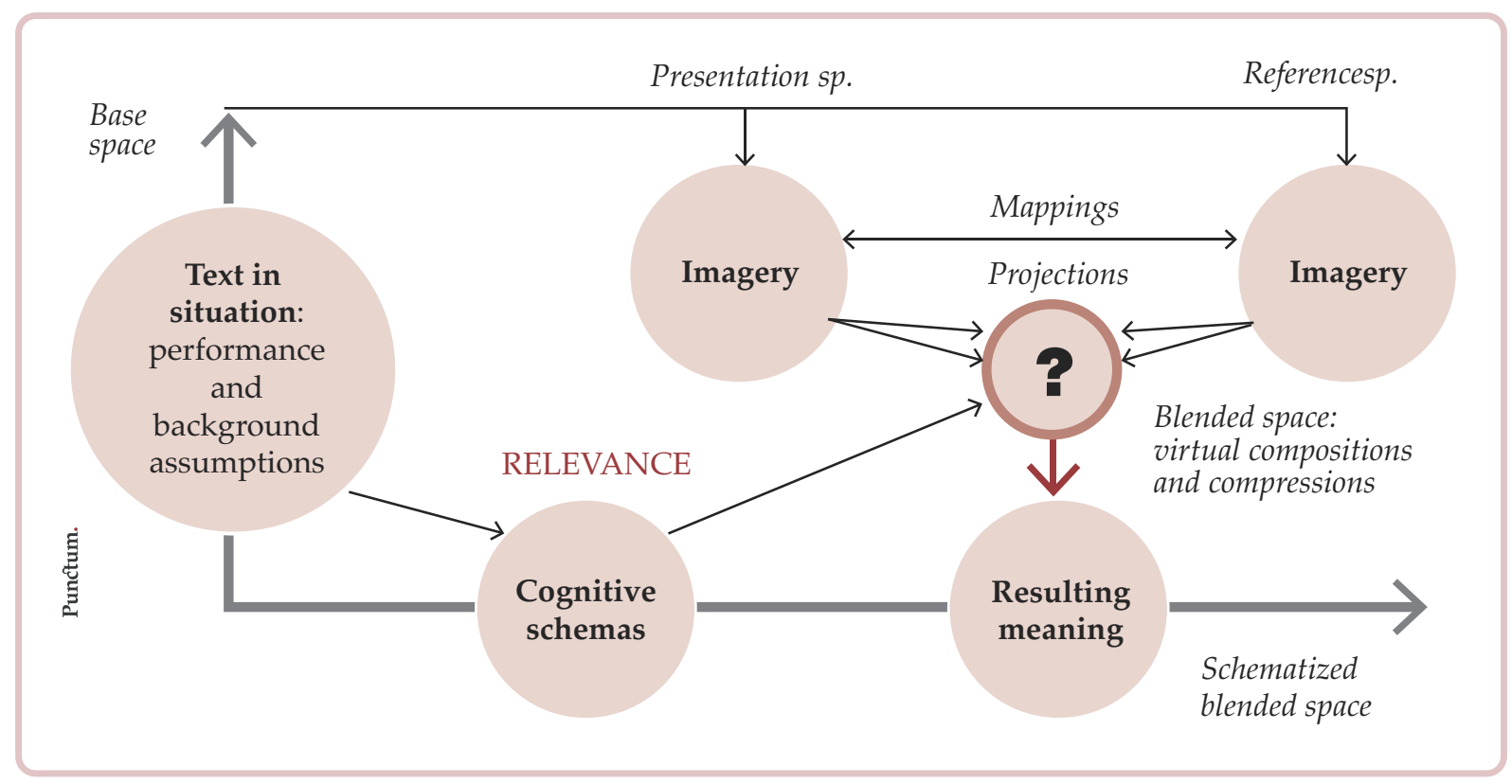

Figure 1. The semiotic blending model (Brandt, 2020, p. 107) 
In the chapter discussing the interpretation of Leonard Cohen's famous song Hallelujah, Brandt (2020) describes his blending model as a flow from a previous existing schematization of meaning to a new schematization emerging from the blending. This movement creates a new meaning space in which all the previous meanings are virtually present and potentially activable for generating interpretation. In other words, sign-complexes constitute polysemy fields activated contextually and stored in personal culture. The problem is that Brandt's model only works at the level of human linguistic communication, namely the production and interpretation of different text genres. This is still a topological model: it is a closed cycle that leads from one schematization to another. How can one introduce transformation over time?

The first step is, of course, the introduction of time, as infinite semiosis presupposes temporality (figure 2). The second element is the introduction of the wholeness and schematization dialectic over time.

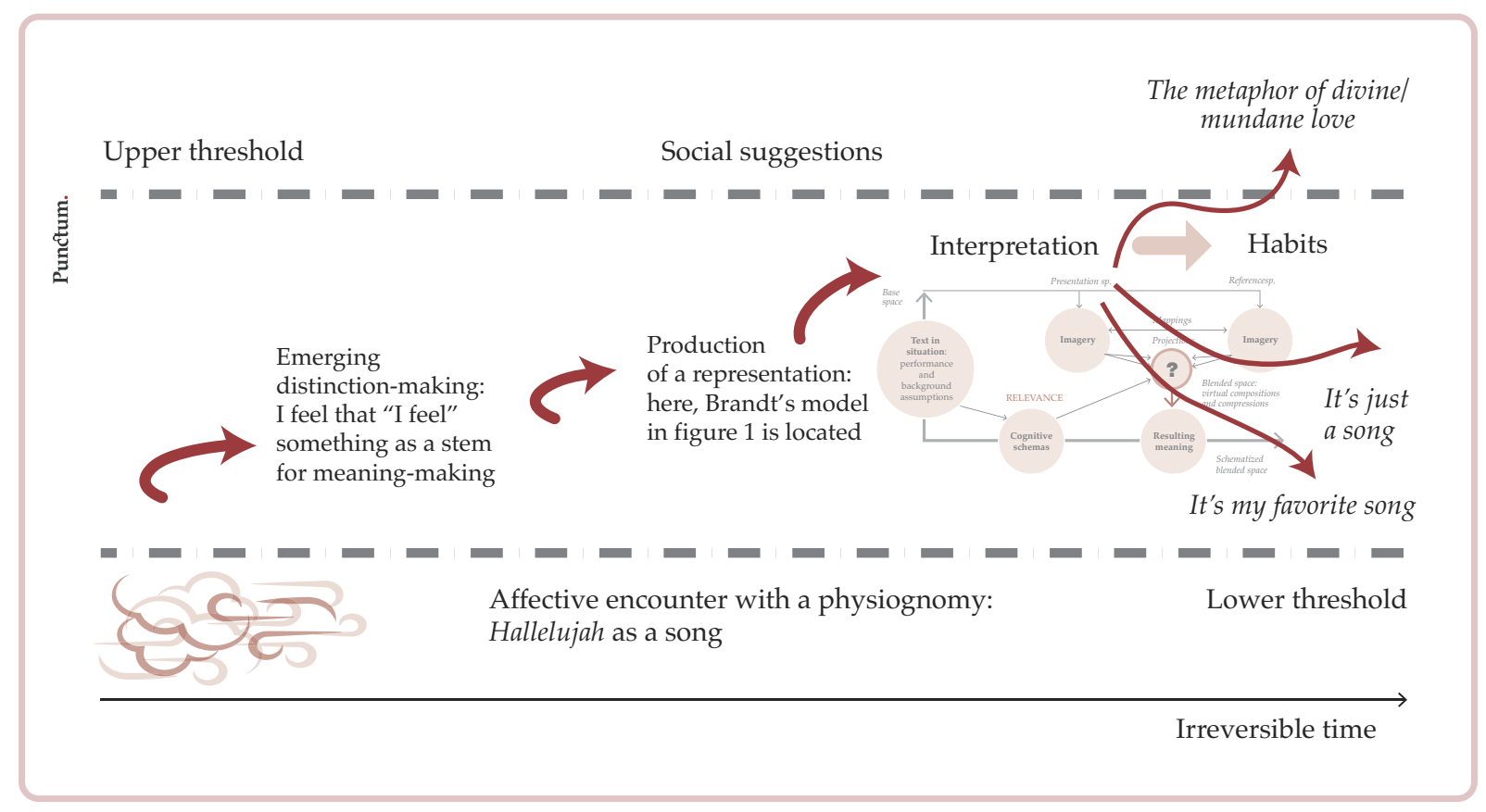

Figure 2. Dynamics of interpretation

In his analysis of the Hallelujah song, Brandt focuses on the linguistic level. Yet, this is not what we primarily experience. Since the first chord, we experience a physiognomy made of several sign-complex elements: we experience a gestalt quality. Indeed, no matter what instrument or performer is playing, we immediately relate to the sign-complex. The recognition of the song is based on this primary affective relating. Yet, recognition is already a semiotic process that calls into question the text's cultural aspects, e.g., the song form, the performative dimensions, the ballad genre, the biblical title, etc. The lower threshold's location between the Firstness of the gestalt quality of the melody and the Secondness of the relationship between me as the audience and the text is pro- 
bably fuzzy. It stems from my affective relation to the material qualities (not necessarily the qualia as distinct elements): I feel that I feel is the stem for the meaning-making. When I position myself as the audience, I create the conditions for an interpretation, and here comes into play Brandt's mental space model. It does not describe the whole process, but a part of it. The unfolding of the process in time implies that the moment after any sign is produced, we also have the production of a constraint, of a habit.

Social suggestions represent another set of constraints, what Brandt calls the reference space, that guide my interpretation in a specific direction. If I accept these suggestions (I am, of course, not obliged to do so), I can, for instance, produce an interpretant like the one suggested by Brandt: the song is a blend of the spaces of meaning referring to the divine and mundane love. Once I produce the interpretant 'love,' I activate a meaning field that is infinite and fuzzy, like all signs referring to human values. This meaning field is characterized by being above the schematization threshold: they are hyper generalized signs (Valsiner 2005). Both the meanings below and above the threshold cannot be represented through Brandt's schematization. Primary affective experience can only partly be schematized (experiencing Hallelujah as an affective gestalt) because the meaning field can only partially be circumscribed in schematic terms. Everybody understands what I mean by 'love,' but we cannot agree on a schematic and finite definition.

The process of interpretation thus builds on top of the levels I have described in figure 2. Moreover, interpretation as an action is partially constrained by the previous chain of interpretations (habits as a framework) but at the same time produces a sign in the immediate future (habit as channeling). Interpretation is not determined; instead, it results from a field of vectorial forces. The outcome of the interpretation process can lead to very different effects in the future. I can comply with the social suggestions as in the example of Brandt's interpretation). I can simply produce a sign that neutralizes the schematization ("it is just a song"), or I can create my version of it and make it my ("My favorite song").

\section{Conclusion}

Per Brant's book is a very productive attempt to design a general cognitive architecture model to account for the meaning-making process. In this sense, Cognitive Semiotics succeeds in creating a consistent model and introducing a precise terminology. However, I think it fails in introducing any radical novelty. The extent to which cognitive semiotics' enterprise moves beyond the existing theoretical reflection in biosemiotics, semiotic cultural psychology, linguistic anthropology, and textual semiotics is somewhat unclear. The attempt to bring a humanistic and holistic perspective into cognitive 
science is relatively unachieved since Bruner's (1990) times. Bruner has already demonstrated that it is impossible to reduce the problem of human meaning to schematization. We need a dynamic and temporal understanding, such as the narrative mode of thought (Bruner 1990). Brandt's model certainly has potential; still, it is a structural model that needs to be inoculated with the temporal dimension. This limitation becomes especially acute when discussing the narrative form (in chapter 9) of the modern novel. His mapping continues to be that of a set of repeated pictures of structural transformations.

The infinite semiosis process is based on the dynamic dialectic of schematization and wholeness, whereas Firstness feeds into Thirdness only to generate a new cycle in irreversible time. Brandt's book and cognitive semiotics seem focused on mapping relationships' configuration while overlooking their dynamic aspect as if cognition was limited to schematization.

\section{References}

Brandt, Per Aage 2020. Cognitive Semiotics: Signs, Mind, and Meaning. London: Bloomsbury Academic.

Bruner, Jerome S. 1990. Acts of meaning. Cambridge, MA: Harvard University Press.

Innis, Robert E. 2016. Between philosophy and cultural psychology: Pragmatist and semiotic reflections on the thresholds of sense. Culture \& Psychology 22 (3): 331-361.

Simmel, Georg 1994. Bridge and door. Theory, Culture $\mathcal{E}$ Society 11 (5): 5-10. https: / / doi.org 10.1177 / 026327694011001002

Tateo, Luca 2018. Affective semiosis and affective logic. New Ideas in Psychology 48: 111.

Valsiner, Jaan 2005. Scaffolding within the structure of dialogical self: Hierarchical dynamics of semiotic mediation. New Ideas in Psychology 23 (3): 197-206.

Zlatev, Jordan 2012. Cognitive semiotics: An emerging field for the transdisciplinary study of meaning. Public Journal of Semiotics 4 (1): 2-24.

\section{AUTHOR}

Luca Tateo Associate Professor, Department of Special Needs Education, University of Oslo, Norway, and Associate Professor, Research Centre for Cultural Psychology, Aalborg University, Denmark. 


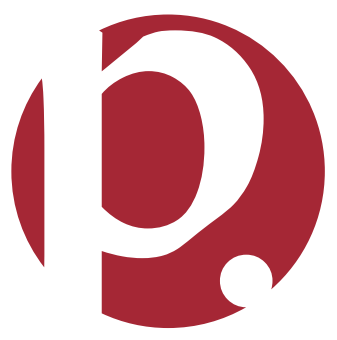

